\title{
A Framework for Monitoring Interface for Local Area Networks via Personal Bluetooth Computing
}

\author{
Deepti.C \\ Assistant Professor \\ Department of Information Science and Engineering \\ The Oxford College of Engineering, \\ Bangalore, India
}

\begin{abstract}
In this paper, an interconnection method between Bluetooth networks/devices and IP based Local Area Network is suggested to make possible LAN monitoring without physical connectivity. A Network Monitoring System (NMS) has been designed which has the role of connecting Bluetooth enabled devices to the Internet Protocol based Local Area Network. The client server based networking model is applied to the system consisting of a personalized Bluetooth network (laptop and mobile) and the specific host on the LAN to be monitored. As a result, an increase in the network error detection capability of the inter-connected network is achieved giving more access and flexibility in LAN infrastructure monitoring.
\end{abstract}

\section{General Terms}

Bluetooth, Security, Surveillance.

\section{Keywords}

Bluetooth, mobile ad hoc network, Network monitoring, OS Linux, client-server architecture

\section{INTRODUCTION}

Open Source based Network Monitoring has become in recent years one of the most demanding services to be provided to local area network infrastructure. The availability of clear-cut information about the operations of a given network allows for the development of many value-added applications. For outdoor applications, GPS or GSM-based monitoring systems offer good performance at reasonable price. Unfortunately they do not work well indoors. This has led to investigations on the use of Bluetooth technology, which is increasingly available in ordinary mobile devices. Their restricted range of transmission allows the circumscription of the area where devices may be found. This paper discusses the development of a cost-effective monitoring system based on the Bluetooth technology, which can reach a precision of about $2 \mathrm{~m}$, and does not require dedicated hardware and installation of additional software in mobile devices. This work makes novel contribution in developing an approach based on inquiries between nearby Bluetooth enabled devices issued at different power levels.

\section{EXISTING METHODOLOGIES}

Both client-server and peer-to-peer architectures are prone to failures. These failures might be as a result of the infringement of network security by way of denial of service attacks or through a natural disaster that can lead to the destruction of a network node. These can lead to the failure of critical services in a network. Hence computer security and management for locally established networks is a key component of any organization's operating and strategic plan. But if a loophole is revealed, there must be procedures in place to identify the occurrence of an "event", to prevent future occurrence and to analyze how the situation was caused. These procedures must have the capability to track the source of the problem and ascertain remedial actions. They can also be used to provide reliable and usable evidence for legal proceedings.

Currently, existing solutions for monitoring Local Area Networks based on open source UNIX style operating systems like Linux have so far based their focus on monitoring systems that require wired networks to permit total connectivity or necessitate the use of expensive WLAN access hardware. Network protocol analyzers using dedicated hardware tools are indeed available on hand which can be connected almost anywhere in the wired network . These analysers are used to inertly observe network traffic. Yet network monitoring [1-4] is not a trivial task and usually requires dedicated hardware or software on the tracked devices, with cumbersome network setup and high deployment costs. There is a requirement for a solution to be developed, which allows for easy unwired access for administration purposes to already existing LAN infrastructure.

This therefore requires an investigation on the use of Bluetooth technology, which is increasingly available in ordinary handheld devices like laptops and mobiles, which have a limited range of action, allowing the circumscription of the area where mobile devices may be found. Some of the applications developed using Bluetooth Ad Hoc networks include the Bluetooth Umbrella Project, BEDD and inter-vehicle communication.

At Trinity College, the Networks and Telecommunications Research Group have built a project called Umbrella net [5] which utilize ad-hoc networks to connect people in urban space. The idea is that temporary networks would be created when people in close proximity open their Bluetooth equipped umbrellas. The umbrellas which house the routers are linked via Bluetooth to PDAs.The BEDD (Bluetooth-Enabled Dating Device) application uses Bluetooth wireless communication to search for "strangers" phones for their personal profiles. The application runs in the background on a phone and exchanges a profile with other phones in range. Sussex University Communications Research Group has used Bluetooth Ad Hoc networks to build a system of inter-vehicle communication [6]. Bluetooth is a good choice for inter-vehicle communication because the nodes (vehicles) frequently move in and out of range of the master node and local piconets.

\section{SYSTEM OVERVIEW}

For limiting cost and system complexity, the following design criteria is adopted:

- Affordability - the system should offer localization services at low costs;

- Modularity - in order to enable change any part easily with cheaper or better performing solutions.

- No dedicated software installed in the monitored devices, so as not to restrict the number of potentially trackable devices. 


\subsection{Implementation approach}

The initial step before implementing wireless network monitoring activities is to establish the needs of the organizational LAN. This process includes consideration about initial expense for software as well as ongoing licensing and support costs, the time available from technical support providers to set up, learn and manage dayto-day installed software, the complexity of the network, anticipated growth in the size, any contemporary issues with network performance, level of reporting required on network availability or usage, and any security issues relating to the network.

\subsection{Review of the implementation}

To confirm that network monitoring is working, any logical map drawn by the planned approach should be compared with the physical map drawn by considering the practically existing resources. It would be worth repeating this process whenever new devices are added to the network. This will ensure that new devices are incorporated in network monitoring activities and that the network monitoring software is able to detect changes to the network topology.

\subsection{Initial design}

At the start of the project, the various ways in which the system could be implemented was considered. Firstly the crucial elements such as how the system should be structured, what parameters should be stored and how these should be stored were considered. Initially the system was considered to be divided into two separate parts:

1. The user interface part, which would be responsible for talking, validating and displaying the settings from-or-to the user.

2. The back-end part, which would be responsible for applying the configuration to the Linux machine.

However, the system was also required to be expandable, enabling extra functionality to be added to it in future which leads to an idea of a modular design approach. The resulting system that was developed comprises four different components (Figure 1):

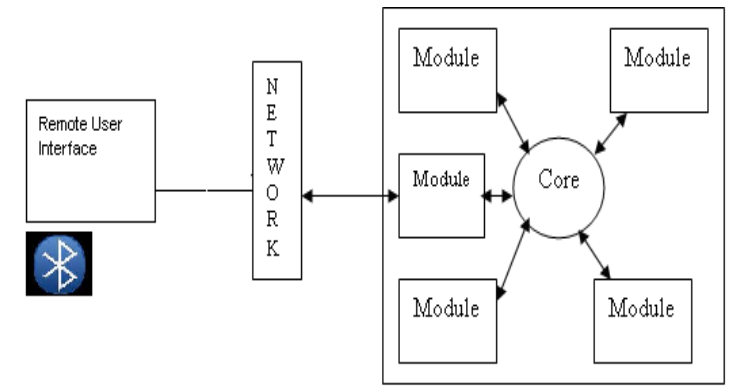

Fig 1: System architecture

(a) An ad hoc network between a Bluetooth enabled device and the Bluetooth enabled host or Interface (b) Bluetooth enabled laptop and mobile (c) Bluetooth stack utilities on the host to be monitored on the LAN and (d) Monitoring and Tracking System.

Bluetooth can be thought of as a replacement for cables of a few meters (USB and legacy interface like serial port, parallel port and PS2 mouse, or a keyboard) for applications that have low bandwidth requirement. Technically, Bluetooth can interconnect up to 7 devices [7] at speed up to $950 \mathrm{kbit} / \mathrm{s}$ and distance up to 10 meters. There are two implementations of Bluetooth stacks under Linux: Bluez and Affix [8]. This paper focuses on the use of Bluez only.
Bluetooth sensing capability for the implementation of a LAN monitoring interface is provided using an external Bluetooth dongle hardware (Here a Bluetooth USB dongle having the pair value "Vendor ID: Product ID" = 0000:0000:0048 has been used) on Open source desktops that are part of a LAN. The Bluetooth sensors on the LAN have the function of connecting to units in the surrounding Bluetooth enabled space, broadcasting inquiry pack connection method at different power levels and listening for responses. The monitoring server is a Bluetooth enabled laptop equipped with Bluetooth stack capability on Debian Linux. Fourteen options were designed to investigate aspects of the performance of the LAN. For the experiments, a hardware test bed consisting of Class 1 and Class 2 Bluetooth modules were set up.Linux-based development boards mentioned above hosted these modules.

The modules of the current Bluetooth LAN monitoring interface have been designed to include display of active hosts in the network, bandwidth usage, memory usage, host computer information, packet information, and a graphical user interface developed using shell programming. The collected responses are sent to the monitoring server together with the identity of the monitored host via an ad hoc network formed by the Bluetooth sensors on the devices themselves. The parameters are displayed and can be stored for accessing current data about the tracked devices.

\section{EXPERIMENTAL RESULTS}

The results of two types of experimental trials are presented below. The first is the description of the time it takes to setup and establish a Bluetooth connection among the 2 static devices participating in the monitoring activity. The second is the categorization of the range in which Bluetooth devices can establish connections and the type of data transfer that is achieved at a given range.

\subsection{Device discovery characterization}

One restraining factor in the amount of data that can be transferred over a Bluetooth connection in local area environment is the amount of time spent discovering other devices and establishing connections.

In this test, one device was set up as an inquirer with the remaining devices (only one at present) on the LAN acting as an inquiry target. It is possible to lay down a timeout on device discovery, but the HCI layer interface fixes this value at multiples of $1.28 \mathrm{~s}$. Each test was tried 25 times. In the case of the $1.28 \mathrm{~s}$ test, it was seen that a minimum of one device is discovered $84.7 \%$ of the time. For the final test, the time taken to search each individual device was measured. The discovery time can tentatively vary between devices if the clocks are not primarily well synchronized, which is the case when devices from different manufacturers are used. It is seen from this experiment that the average connection time to an individual Bluetooth device around $2 \mathrm{~s}$. Furthermore, there are no much variations between different Bluetooth devices.

\subsection{Range and Throughput}

A second limiting factor for the appropriate use of Bluetooth in mobile environments is its performance at significant distances. Two aspects of this performance are important here: the distance over which devices can set up and sustain connections and the data transfer possible at the given distance. The first factor measures the range at which two Bluetooth devices can effectively discover one another and establish a connection. The 
second explores the data transfer that can be achieved at distances within the maximum range established by the first experiment. During these experiments, the Bluetooth devices were in an outdoor, line-of-sight environment far from any likely interference. The throughput tests were conducted in this setup by repetitively trying out FTP transfers between connected devices.

The first task in characterizing Bluetooth's performance over large distances was determining the maximum range over which devices can discover one another and establish connections. Table 1 lists the results of basic range tests for the two highest power classes of Bluetooth devices.

Table1. Range for different power classes

\begin{tabular}{|c|c|c|c|}
\hline $\begin{array}{c}\text { Device } \\
\text { Power Class }\end{array}$ & $\begin{array}{c}\text { Max Output } \\
\text { Power } \\
(\mathrm{mW})\end{array}$ & $\begin{array}{c}\text { Max Output } \\
\text { Power } \\
(\mathrm{dBm})\end{array}$ & $\begin{array}{c}\text { Expected } \\
\text { Range } \\
\text { (Metres) }\end{array}$ \\
\hline Class 1 & 100 & 20 & 100 \\
\hline Class 2 & 2.5 & 4 & 10 \\
\hline
\end{tabular}

These results are surprising in that both classes far outperform the minimum ranges required by the Bluetooth specification. This over-performance is a very encouraging indication of Bluetooth's feasibility for use in mobile and LAN environments for the purpose of monitoring. The stability of the data rates over a large range of distances is also encouraging.

Throughput is the maximum rate at which frames or packets can be transmitted from the source to the destination with zero loss of data or errors which are measured in bits per second (BPS). It should be noted that the physical interface for the devices used in the experiments is restricted to a data rate of $115.2 \mathrm{kbps}$, a constraint common to nearly all currently available Bluetooth devices. Also, Bluetooth automatically incorporates FEC coding (at a $2 / 3$ rate) during the transmission of packets [9]. These two factors clarify the limited maximum throughput realized in the experiments as shown in Figure 2.

While the realized throughput is significantly less than Bluetooth's rated maximum, it can be believed that the trends observed in this work will hold for higher data rate Bluetooth devices.

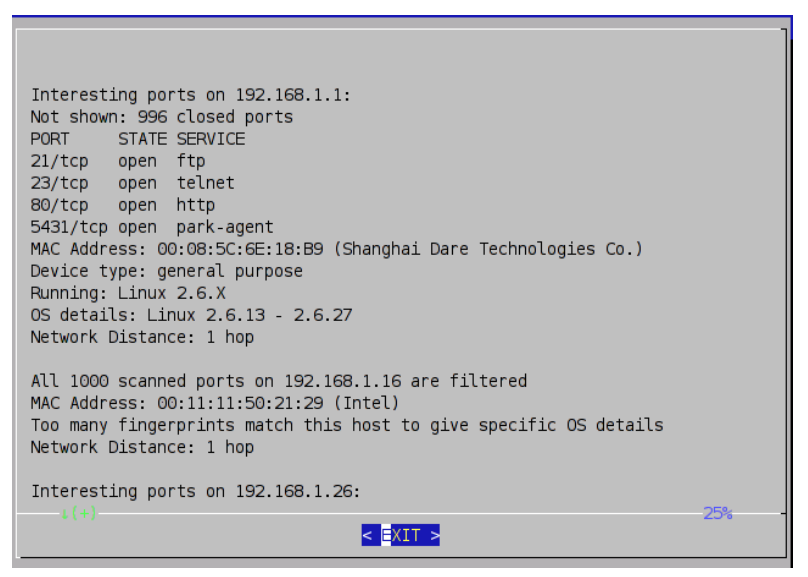

Fig 2: All hosts on the LAN

\section{RELATED WORK}

Several studies have investigated the use of wired and wireless technologies for the creation of an indoor monitoring service. Some of them deal with simulated systems only, based on theoretical data supplied from existing Ethernet specifications, or they make an evaluation of wireless technologies in a general monitoring platform [10]. Others are based on experimental tests [11-12] using RSSI and reachability data. In some cases RSSI values [13] obtained are very different from the data obtained through this work and this is mostly due to the context in which measures are taken (class of the devices and propagation characteristics). None of these works, however, takes sufficient consideration of delays introduced by Bluetooth sensors when measuring RSSI, which can be very high and make the approach inapplicable.

For applications requiring a lot of precision such as robot control, other technologies can be more appropriate. For example timesynchronised transceivers with carrier-phase point positioning [14] may obtain sub-centimetre precision; infrared and ultrasonic signals achieve an accuracy of about $15 \mathrm{~cm}$. However, the application field of these technologies is completely disjointed from the target of context or location of the applications, where Bluetooth is well suited. Examples are systems for giving directions in large buildings like airports or hospitals, or for offering context-sensitive pieces of information in museums or department stores. These applications, in fact, can tolerate a lower accuracy, but their effectiveness and diffusion is highly dependent from widely available positioning infrastructures, which inevitably must be cost effective.

\section{CONCLUSION}

The need of users to share contents and to access network application services whenever and anywhere are increasing, as users have high performance personal devices which operate in advanced network communication environments. In this paper, an interconnection method between IP based network and Non-IP based Bluetooth Personal Area Network (PAN) is presented. Adhoc network connection protocols are applied in order to virtualize personal devices and Bluetooth devices as an overlay network and to provide users with the service to share network information through a seamless connection between authorized personal devices. Since Bluetooth devices are characterized as being particularly decentralized and self-organized, the monitoring system was also designed to allow Bluetooth devices to connect and communicate with a peer. The connected adhoc network is composed of hosts that propagate inquiry advertisement and hosts that relay messages, index advertisements of devices and service information from hosts in order to let users discover them.

\section{ACKNOWLEDGEMENTS}

Thanks to my professors and peers at the R\&D Lab, Department of Computer Science at DSCE, Bangalore. Thanks to Prof. Chandrasekharan K.A of Christ University for reviewing the paper.

\section{REFERENCES}

[1] Siddhartha Bunga and Tilman Wolfy, "A Characterization of High-Performance Network Monitoring Systems and Workloads", Cisco Systems, Inc., San Jose, CA, Department of Electrical and Computer Engineering, University of Massachusetts, Amherst, October 2005

[2] W. Wu, M. Crawford, "The Performance Analysis of Linux Networking - Packet Receiving”, 2005 
[3] Image Stream Internet Solutions," Network MonitoringWhite Paper"

[4] A.Kajackas, E.Šilanskas, A. Šaltis, "Customer Premises Local Area Network: Concept and Problems", Vilnius Gediminas Technical University, Vilnius, Lithuania, December 2001

[5] F. Forno, G. Malnati and G. Portelli, "Design and implementation of a Bluetooth ad hoc network for indoor Positioning", October 2005

[6] Patric Murphy, Erick Welsh, and J. Patric Frantz, "Using Bluetooth for short-term Ad Hoc connections between moving vehicles", IEEE, VTC, pp.414-418, 2002

[7] R. Gu'erin, J. Rank, S. Sarkar and E. Vergetis, "Forming Connected Topologies in Bluetooth Adhoc Networks", University of Pennsylvania ,July 2002

[8] P. Johansson, M. Kazantzidis, R. Kapoor, M. Gerla, "Bluetooth: an enabler for personal area networking", IEEE Network, Sep/Oct 2001, Vol.15, No. 5, pp. 28-37

[9] Bluetooth SIG, "Specification of Bluetooth system, ver 1.1"
[10] Jens Andreasson, Mikael Ekström, Ali Fard, Javier Garcia Castano, Tord Johnson, "Remote System for Patient Monitoring Using Bluetooth." Proceedings 1st IEEE International Conference on Sensors, Orlando, Florida, June 2002 , pages $304-307$.

[11] T. Salonidis, P. Bhagwat, L. Tassiulas, and R. LaMaire, "Distributed Topology Construction of Bluetooth Personal Area Networks", Proceedings of the IEEE INFOCOM 2001,(Anchorage, Alaska, April 2001) 1577-1586.

[12] Kiran Thapa, Steven Case," An Indoor Positioning Service for Bluetooth Ad Hoc Networks", Department of Computer \& Information Sciences, Minnesota State University, Mankato, May 2002.

[13] Byeong-Thaek Oh, Sang-Bong Lee, Ho-Jin Park, “An Interconnection Method between Bluetooth Network and P2P Network", Real-Sense Media Convergence Research Team, Electronics and Telecommunications Research Institute, Daejeon, Korea, ICACT February 2009

[14] Osok Song*, Chaegwon LimO, and Chong-HoChoi, "Mobility Management in Bluetooth ad hoc networks", 2004 